\title{
Equidad, género y matemáticas en la escuela mexicana
}

Equity, Gender, and Mathematics in Mexican Schools

Equidade, gênero e matemáticas na escola mexicana

\author{
Sonia Ursini* (iD o000-0002-3593-8854 \\ Martha P. Ramírez-Mercado** (iD) 0000-0002-6652-5585
}

\begin{abstract}
Artículo de reflexión
Revista Colombiana de Educación, N.7 73. Segundo semestre de 2017, Bogotá, Colombia.

doi: 10.17227/01203916.73rce211.232

Para citar este artículo: Ursini, S., y Ramírez, M. (2017). Equidad, género y matemáticas en la escuela mexicana. Revista Colombiana de Educación, (73), 213-234.
\end{abstract}

\section{(ल) $(\mathbb{1} \Theta$}

Recibido: 01/02/2017

Evaluado: 17/03/2017 


\title{
Resumen
}

A partir del análisis de entrevistas realizadas en Ciudad de México a profesores de primaria y secundaria, así como a estudiantes de tercero de secundaria, se pone en evidencia su visión de género y equidad con relación a la enseñanza y el aprendizaje de las matemáticas. Identificamos algunos factores que propician y fortalecen la inequidad de género en matemáticas: el tipo de interacciones diferenciadas por género que los docentes establecen en la clase; las creencias de que las matemáticas son un dominio masculino; las estrategias de compensación que diseña el profesorado con la intención de propiciar la equidad de género; las expectativas de aprendizaje de las matemáticas diferenciadas por sexo que tiene el profesorado; los estereotipos de género de los padres de familia y del profesorado. La presencia de estos factores se ilustra mediante extractos de entrevistas. Se concluye señalando que si bien es necesario garantizar formal y legalmente el acceso igualitario de hombres y mujeres al conocimiento matemático, esto no es suficiente para lograr la equidad. Consideramos esencial darse cuenta de que para alcanzar una mayor equidad es necesario que la sociedad en general y la comunidad escolar en particular superen los estereotipos de género, todavía profundamente arraigados, que conducen a tener expectativas diferenciadas por sexo con relación al aprendizaje y el uso profesional de las matemáticas. Se requiere trabajar enérgicamente en esa dirección.

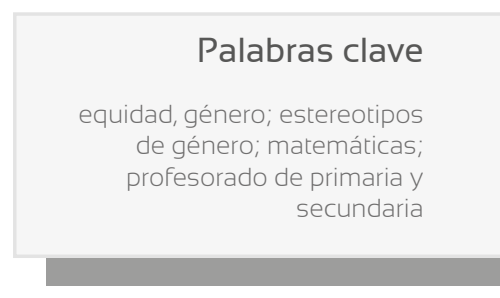

\section{Keywords}

equality; gender; gender

stereotypes; mathematics; primary

and secondary school teachers

\begin{abstract}
From the analysis of interviews carried out in Mexico City with primary and secondary school teachers, as well as with third-year students, their gender and equity vision in relation to the teaching and learning of mathematics is highlighted. We identify some factors that propitiate and strengthen gender inequality in mathematics: the type of interactions differentiated by gender that teachers establish in class; the belief that mathematics is a male domain; compensation strategies designed by teachers aiming to promote gender equality; teachers' expectations about learning mathematics based on gender; parents' and teachers' gender stereotypes. The presence of these factors is illustrated by excerpts from interviews. We conclude that, while it is necessary to formally and legally guarantee the equal access of men and women to knowledge, this is not enough to achieve equality. It is essential to realize that, in order to achieve greater equality, it is necessary for society in general and the school community in particular to overcome the still deeply ingrained gender stereotypes that lead to expectations based on gender regarding the learning and professional use of mathematics. Strong work is required in that direction.
\end{abstract}

\section{Resumo}

A partir da análise de entrevistas realizadas na Cidade do México a professores do ensino básico e secundária, assim como a estudantes de terceiro ano de secundária, evidencia-se sua visão de gênero e equidade em relação ao ensino e a aprendizagem das matemáticas. Alguns fatores que propiciam e fortalecem a inequidade de gênero nas matemáticas foram identificados: o tipo de interações diferenciadas por gênero que os docentes estabelecem durante a aula; as crenças de que as matemáticas são um domínio masculino; as estratégias de compreensão que desenvolve o professorado com a intenção de propiciar a equidade de gênero; as expectativas de aprendizagem das matemáticas diferenciadas por sexo estabelecidas pelo professorado; os estereótipos de gênero dos pais de família e do professorado. A existência desses fatores é ilustrada mediante extratos das entrevistas. Como conclusão, bem se é preciso garantir formal e legalmente o acesso igualitário de homens e mulheres ao conhecimento matemático, isso não é suficiente para conseguir a equidade. É vital perceber que para conseguir uma maior equidade é preciso que a sociedade em geral e a comunidade escolar superem os estereótipos de gênero, ainda profundamente arraigados, que são causa das expectativas diferenciadas por sexo em relação à aprendizagem e o uso profissional das matemáticas. É necessário trabalhar energicamente nesse propósito.

\section{Palavras chave}

equidade; gênero; estereótipos de gênero; matemáticas; professorado de básica e secundária 
En las últimas décadas hemos asistido a un notable incremento, en distintos ámbitos, del uso de las palabras género y equidad. Estas dos palabras aparecen con frecuencia en discursos oficiales, en distintos tipos de debates, en declaraciones de organismos internacionales y gubernamentales, en artículos periodísticos. Sin embargo, no siempre su significado queda explícito, lo que se presta a interpretaciones subjetivas y a menudo no compartidas. En el ámbito académico la problemática de "género" y "equidad" se estudia en relación con distintas disciplinas y es aquí donde se busca definir con más precisión estos dos términos.

\section{Identidad de género y matemáticas}

La palabra género a menudo se usa como sinónimo de sexo, si bien, en las últimas décadas se ha ido precisando su significado principalmente desde los campos de la sociología, la antropología, la psicología y los estudios feministas (por ejemplo: Barbieri, 1996; Burton, 1986, 1990; Bustos, 1994,2001; Flores-Palacios, 2001; Lamas, 1986; Rascón, 2000; Scott, 1986; Spencer, 1993; Subirat, 1997; Tiedemann, 2002). Se ha señalado que género y sexo no solo no son sinónimos, sino que mientras el sexo de un ser humano es una característica biológica, el género es una construcción sociocultural, elaborada a partir de las diferencias biológicas. De la misma manera se han ido delimitando histórica, cultural y socialmente los roles que hombres y mujeres tienen que asumir y desempeñar en una sociedad determinada. Nacemos, por lo tanto, con un sexo determinado, pero no con un género determinado. La idea diferenciada de género y la identidad de género se van construyendo socialmente, a través de la educación que recibimos desde edades muy tempranas en la familia, en la escuela, a través de los medios, de los juguetes y de los juegos, de las relaciones sociales que se establecen en entornos distintos.

Hombres y mujeres aprendemos a comportarnos, a sentir y pensar de manera diferenciada de acuerdo a los patrones que dominan en el entorno social, cultural y económico en el que vivimos. Ser hombre o ser mujer en cierta sociedad y en un determinado momento histórico se aprende y aprehende al aceptar ejercer ciertos roles, diferenciados por sexo, que se valoran en consonancia con la economía y las decisiones políticas que predominan en una sociedad determinada.

La identidad de género está conformada por las actitudes, los valores, las creencias, las normas, el comportamiento y la representación social de sí misma que tiene una persona en relación con los objetos, situaciones y exigencias que provienen de los entornos social, cultural, económico y político. Las mujeres y los hombres se van educando de manera distinta para que adquieran creencias, valores, normas y patrones de comportamiento 
diferenciados, elaborados y transmitidos por el entorno en el que viven. En una misma sociedad estas características pueden ser compartidas por grupos más o menos numerosos de mujeres u hombres, si bien no necesariamente un solo grupo abarca a todas las mujeres o a todos los hombres. Se van creando así sentimientos de pertenencia a cierto grupo y se va construyendo la identidad de género. Además, se van construyendo también otras facetas, no necesariamente compartidas por la mayoría, que pueden ser el reflejo de la clase social de origen o pertenencia, de la educación, la cultura, las normas, los valores, las creencias dominantes en cierto grupo social (la comunidad en la que se vive, la Iglesia, la familia, la escuela, la etnia, la profesión, entre otras). Todo ello influye en la conformación de la identidad que agrupa entre sí a ciertas personas y las distingue de otras, sean hombres o mujeres, así como en la conformación de la identidad de género.

Un elemento que contribuye notablemente en la construcción de la identidad y, en particular, de la identidad de género, lo constituye la educación e información que se recibe en la escuela junto con las relaciones que se establecen en el salón de clase entre profesor y alumnado. Desde los primeros niveles de educación básica el profesorado va fomentando la formación de ciertas creencias, actitudes, valores, comportamientos y la construcción de determinados estereotipos y, en estos procesos, va proyectando su propia visión de género. En particular, esta se manifiesta a través de la enseñanza de las materias curriculares. Es muy común, por ejemplo, que el profesorado tenga una visión de género asociada a ciertas asignaturas, como, por ejemplo, las matemáticas y la lengua. Está comprobado que la gran mayoría del profesorado considera que las niñas son mejores para la lengua y las materias humanísticas, mientras los varones lo son para las matemáticas, la ciencia y la tecnología, dado que se considera que estas últimas requieren un pensamiento más lógico y racional que, supuestamente, no caracteriza al sexo femenino (Sherman y Fennema, 1978; Burton, 1986; Ernest, 1980; Flores, 2007; González, 2003; Hyde, Fennema y Lamon, 1990; Ramírez, 2006; Rivera, 2003; Ursini, 2004). Este, junto con otros estereotipos de género presentes en muchas sociedades actuales, se van transmitiendo y reforzando a lo largo de la educación que reciben niños y niñas en la escuela y van contribuyendo a la formación de la identidad de género, diferenciada por sexo, en relación con las distintas asignaturas. Por lo tanto, esta deviene también una de las funciones de la educación escolar, junto con la transmisión de saberes, valores, normas y conductas.

La identidad de género diferenciada que se va formando respecto a las matemáticas no ha sido, sin embargo, muy estudiada y poco sabemos todavía acerca de cómo influye en el desempeño y acercamiento a esta disciplina de alumnos y alumnas (Ursini, 2013). A pesar de ello, hay evidencia -como se ilustrará más adelante- del papel que tiene el profesorado en su construcción. 


\section{Equidad y educación}

Para hablar de equidad y matemáticas, es necesario, ante todo, mencionar algunas posturas relativas a qué se entiende por equidad en educación. A partir del trabajo de Rawls (1979), que discute la equidad educativa desde la teoría de la justicia, se han generado múltiples debates alrededor del tema. Ante todo, se ha señalado que no es lo mismo hablar de equidad que de igualdad. La igualdad, como afirma Bolívar (2005), se refiere a que cada individuo tenga las mismas oportunidades formales. La equidad se refiere a algo mucho más complejo, ya que, si bien se sigue considerando que todos los miembros de una sociedad deben tener las mismas oportunidades para poder acceder, por ejemplo, al conocimiento, al trabajo o, en general, a lo que esta ofrece a sus integrantes, se pone énfasis en la necesidad de tener en cuenta las diferencias individuales y de grupo. Al respecto Rivas (2004), por ejemplo, afirma que para lograr la equidad es necesario apoyar en mayor medida los grupos más vulnerables y desfavorecidos. Pero otros señalan que, ante todo, es necesario aclarar qué se entiende realmente por igualdad de oportunidades y precisar con relación a qué. Como señala Bolívar (2005), han surgido distintos enfoques al respecto, y se distinguen esencialmente por la postura ideológica de los proponentes. Hay quien opina que se podría lograr una mayor equidad si se considerara la posibilidad de usar contenidos curriculares diferenciados con el fin de apoyar más eficazmente los grupos menos favorecidos a alcanzar sus propios intereses (Connell, 1997) y quien, como Guarro (2002), aboga por una base de conocimientos y cultura común para todos, en aras de poder ejercer una ciudadanía responsable.

Ha habido distintas propuestas para lograr una mayor equidad en la educación académica. La mayoría proviene de países que ya han dejado atrás el analfabetismo, donde la escolaridad básica, que comprende la educación elemental y parte de la educación media-superior, abarca realmente toda la población, y las desigualdades sociales no son tan pronunciadas. Este no es el caso, por ejemplo, de la mayoría de los países de América Latina, donde sigue habiendo población analfabeta, la educación básica no siempre llega a todos y existen diferencias sociales y económicas muy pronunciadas. Las realidades son, por lo tanto, muy diferentes, lo que requiere un análisis local para, en consecuencia, formular posibles propuestas tendientes a propiciar una mayor equidad en el campo de la preparación académica. Para muchos países de América Latina, en particular para México, como veremos más adelante, la escuela sigue siendo un factor fundamental en la reproducción y el mantenimiento de la diversidad, entre ellas, la inequidad de género. Si bien, como en el caso de México, existen marcos jurídicos que establecen que todos los ciudadanos deben contar con las mismas oportunidades de acceso y permanencia en

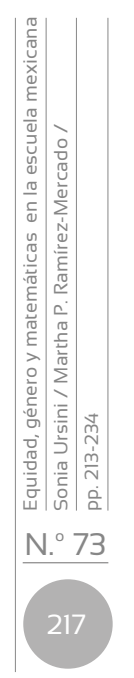


el sistema educativo nacional (Ley General de Educación, arts. 2. ${ }^{\circ}$ y 8. , 1993), no existen las condiciones sociales ni económicas que propicien las mismas oportunidades de aprendizaje para toda la población. Además, como lo ponen en evidencia distintas investigaciones (Ramírez, 2006; Ursini, 2009) a través de la educación escolar se siguen reproduciendo los estereotipos de género y la discriminación hacia las mujeres, lo que fortalece la inequidad de género.

En el campo de la educación matemática, a partir de los años setenta se abordó el problema de la equidad y en particular de la equidad de género. Este tipo de estudios, desarrollados en su mayoría en los países anglosajones, fueron motivados inicialmente por las diferencias en los niveles de logro matemático. Si bien en un primer momento se usaron sobre todo acercamientos de tipo cuantitativo, muy pronto se incorporaron acercamientos cualitativos y se fue dando mayor peso a factores psicológicos, socioculturales y a la idea de justicia social (Fennema, 1974; Fennema y Sherman, 1978; Rossi Baker, 1981). Se investigaron distintas líneas temáticas, como señala Wrigley (1992):

» educación matemática y género desde una perspectiva histórica, con énfasis en el rol del Estado;

» diversidad social y el tipo de control que se genera en el aula, observando las interacciones que ocurren dentro de la misma en las clases de matemáticas;

» la relación familia-escuela, donde se analiza cómo los ámbitos familiares y escolares se intersecan en una multiplicidad de caminos para condicionar la clase social y el género;

» las intervenciones pedagógicas dirigidas a las chicas con el propósito de incrementar la participación de las mujeres en matemáticas.

A partir de estos estudios se buscó identificar posibles causas de las diferencias de género encontradas y se identificaron algunos factores (Hanna, 2003) que propician la inequidad, , por ejemplo:

» el tipo de intercambios diferenciados por género que se establecen entre el alumnado y el profesorado de matemáticas;

» la influencia que ejercen los estereotipos de género de los padres de familia;

» la creencia de que las matemáticas son esencialmente un dominio masculino;

» la creencia de que las matemáticas son útiles sobre todo para la vida futura de los varones. 
Los resultados obtenidos en esos estudios lograron impactar en ocasiones las políticas educativas, sobre todo en algunos países anglosajones y europeos. De este modo se llegó a considerar que la inequidad de género, vista como nivel de logro, ha ido disminuyendo paulatinamente, pero de manera importante, y está casi superada en varios países, si bien, la inequidad persiste todavía en cuanto a la presencia de la mujer en ciertas disciplinas científicas como matemática, física e ingeniería, donde sigue predominando la presencia masculina (Hanna, 2003).

¿Qué tanto los factores arriba señalados como causa de inequidad de género con respecto a las matemáticas y su aprendizaje, están presentes en países con características socioculturales y económicas distintas? ¿Existen, además de los factores ya señalados, otros igualmente importantes, particulares de cada país? Este tipo de preguntas obliga a realizar estudios locales que pongan en evidencia los estereotipos de género que dominan en una sociedad determinada y, en particular, en los espacios académicos y escolares para ver su repercusión en la equidad de género. Si bien ha habido avances, la mayoría de los países están lejos de lograr condiciones de equidad de género tanto en la sociedad en general como en los ámbitos académico y escolar. A continuación, damos un ejemplo que ilustra la falta de equidad de género que todavía se vive en el ambiente académico en México, donde, a pesar de que las mujeres tienen cada vez más presencia, siguen sufriendo presiones que les exigen perfección y eficiencia en distintos ámbitos que van desde el académico al familiar.

En unas entrevistas realizadas en 2013 (Chávez, 2013) a un grupo de académicas mexicanas con relación a su percepción de los avances logrados por las mujeres en los campos científicos, una doctora en química -que hubiera podido ser de cualquier disciplina-comentó que en el pasado no se consideraba que las mujeres pudieran desarrollarse en esta área, pero hoy en día esto ya había sido superado y eran muchas las mujeres que lograban ser aceptadas como investigadoras, lo cual, claro, implica "sortear las actividades familiares y profesionales, hacer bien el trabajo científico y sentirse satisfecha". En este comentario la académica resalta, por un lado, haber tenido la oportunidad de estudiar y de ejercer una carrera científica (un avance sin duda notable en comparación con generaciones anteriores), pero también queda de manifiesto que las exigencias profesionales no tienen en cuenta las "actividades familiares" que en nuestras sociedades se asumen como propias de las mujeres, por ejemplo el cuidado de los hijos, del hogar y de los mayores. Cómo conciliar estos deberes con las exigencias profesionales se considera un problema estrictamente personal, que debe resolverse a nivel individual. Este es un ejemplo de la falta de equidad de género con relación al ejercicio de una profesión académica. En el ejemplo podemos observar cómo la identidad

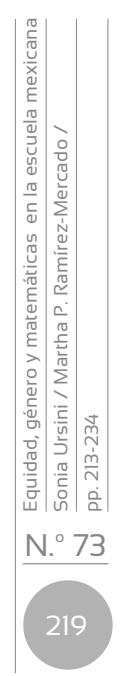


de género (conformada por las creencias, los valores, las actitudes, las normas y los comportamientos con relación a cierto objeto y/o situación), socialmente aprendida, Ileva a la académica entrevistada a no cuestionar en absoluto el hecho de que tiene que respetar la normas, preestablecidas, para poder ejercer y mantenerse activa en su profesión, igual que cualquier varón, sino, además, hacerse cargo de los asuntos familiares, por lo que, como ella comenta, "hay que sortear" entre una y otra cosa; "ese es el reto que hay que vencer para lograr el respeto y la aceptación". Se acepta así la situación, se trata de asumirla como un reto considerando que hay que sentir satisfacción por los logros y, cómo comenta otra académica en esa misma entrevista "agradecer que nos dan la oportunidad, ya que hace algunos años no era asi".

Lo anterior pone en evidencia que tanto la identidad diferenciada de género como la falta de equidad de género, que se han "naturalizado" e "invisibilizado", permean profundamente nuestras sociedades y son sustentos esenciales para su funcionamiento. Como lo señala Emmelhainz (2016)

»El modelo de familia nuclear neoliberal exige que las mujeres, además de contribuir con su sueldo para los gastos de la casa, se ocupen de ella y que eduquen a los hijos... Las mujeres "liberadas" de la clase media y alta sufren la presión en tres frentes donde se les exige eficiencia y perfección: familia, ámbito profesional y normas de género... (2016, p. 227).

La aceptación de esta situación es el resultado de un largo proceso de enseñanza que inicia desde la infancia, en el seno familiar primero, y se va formalizando y legalizando a partir del ingreso a la escuela. Es así como las diferencias de género poco a poco se van formando y consolidando y se reflejan en las creencias que cada individuo tiene acerca de sí mismo, las propias obligaciones, capacidades y limitaciones.

\section{Estudios de género y matemáticas en México}

Los estudios de género y matemáticas en México tienen poco más de dos décadas y la gran mayoría se ha enfocado en los niveles de educación primaria y secundaria. Se ha indagado acerca de logros, actitudes, autoconfianza, el impacto de la tecnología, se han analizado las relaciones que se establecen en el salón de clase entre profesorado y alumnado, así como la visión de los padres de familia. Se han usado distintos acercamientos: cuantitativos, cualitativos y mixtos. Los estudios mostraron que no había diferencias de género estadísticamente significativas en el desempeño matemático debido, esencialmente, a que la gran mayoría del estudiantado tenía logros muy bajos (González, 2003; Rivera, 2003; Real y Ursini, 2010). Sin embargo, las observaciones en clase, las entrevistas individuales 
y grupales pusieron en evidencia diferencias de género muy profundas, sobre todo en relación con la autopercepción del alumnado frente a las matemáticas. Si bien, en general, la auto-confianza va disminuyendo a lo largo de la adolescencia, se observó que la de las niñas suele ser más negativa que la de los varones (Campos, 2006; Eudave, 1994; Morales, 1998; Ursini, 2010; Ursini, Sánchez, Orendain y Butto, 2004; Ursini, Ramírez y Sánchez, 2007; Ursini y Sánchez, 2008). Asimismo, se puso en evidencia el papel del profesorado y de los padres de familia en la formación de estas diferencias y su impacto en la inequidad de género.

Se encontró también una fuerte tendencia a percibir las matemáticas como un dominio masculino, tanto entre los estudiantes de secundaria como entre el profesorado y los padres de familia. Ursini y Sánchez (2008), por ejemplo, resaltan el vínculo que los estudiantes establecen entre inteligencia y matemáticas y la forma diferenciada que tienen de posicionarse ante ello. Mientras los varones consideraban que las matemáticas eran más fáciles y aptas para ellos por el hecho de ser varones y tener, por naturaleza, la inteligencia necesaria para estudiarla, las mujeres asumían que carecían de recursos intelectuales adecuados y que para tener logros aceptables tenían que esforzarse mucho y obedecer las indicaciones del profesor. Una percepción similar se puso en evidencia al analizar las relaciones diferenciadas que establece el profesorado en la clase de matemáticas entre niños y niñas (Ramírez, 2006) así como al entrevistar a algunos padres de familia (Ursini, 2009).

También se indagó el impacto de la tecnología cuando se introduce como apoyo para la enseñanza y el aprendizaje de las matemáticas. Al analizar su uso en secundarias públicas, Ursini y Sánchez (2008) encontraron que podía propiciar el desarrollo de una actitud más positiva hacia las matemáticas, sobre todo entre las niñas, y que junto con actividades guiadas tendientes a fortalecer el trabajo de grupo y las discusiones grupales, se podían modificar ciertos patrones culturales que llevaran hacia una mayor equidad de género. En otro estudio Rodríguez y Ursini (2008) analizaron la relación que establece el profesorado con la tecnología cuando se le pide utilizarla para apoyar sus clases de matemáticas. Encontraron que los profesores varones se enfocaban esencialmente en mejorar su propio dominio del medio, estableciendo así una relación más pragmática y egocéntrica con el uso de la tecnología; en contraste, las profesoras mujeres tendían a usarla para explorar e investigar conceptos matemáticos con el propósito de buscar alternativas viables para apoyar a sus estudiantes a alcanzar mejores logros.

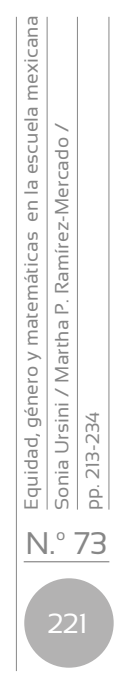




\section{Factores que propician inequidad de género en matemáticas}

Algunas de las investigaciones realizadas en México han puesto en evidencia algunos factores que contribuyen a crear y mantener la inequidad de género con relación a la matemática escolar. Parte de estos factores coinciden con los ya señalados por Hanna (2003), pero identificamos otros a los cuales es necesario prestar atención por su fuerte influencia, a saber:

» el tipo de interacciones diferenciadas por género que establece el profesorado en la clase de matemáticas;

» los estereotipos de género de los padres de familia;

» la creencia de que las matemáticas son un dominio masculino;

» la creencia de que las matemáticas son útiles sobre todo para la vida futura de los varones;

» las estrategias de compensación que diseña el profesorado con la intención de propiciar la equidad de género;

» las expectativas de aprendizaje de las matemáticas, diferenciadas por género, que tiene el profesorado;

» los estereotipos de género del profesorado.

A continuación, presentamos algunos ejemplos que ilustran la presencia de estos factores.

\section{Un ejemplo en la escuela primaria}

Como parte de una investigación más amplia realizada en primarias, Ramírez (2006) entrevistó a catorce maestras con el propósito de conocer su visión de las matemáticas, la confianza en su propio conocimiento matemático y cómo perciben el desempeño matemático de sus alumnos. Los resultados mostraron que todas ellas perciben las matemáticas como una asignatura de difícil acceso, de alto grado de dificultad y que su conocimiento proporciona acceso a mayores y mejores estatus educativos, laborales, familiares y sociales. A pesar de ello, la mayoría posee una baja confianza en su propio desempeño matemático y considera que las matemáticas son esencialmente un campo de dominio masculino.

En relación con la percepción del desempeño matemático de sus alumnos se encontró que establecen una diferencia entre talento y habilidad matemática. Definieron al talento como la capacidad natural y genética para llevar a cabo una tarea; y la habilidad como la capacidad para llevar a cabo una tarea que se desarrolla a través del trabajo, la práctica y/o la ejercitación. Lo relevante de esta distinción es la relación que establecieron entre estos dos términos y los niños y las niñas. Las catorce docentes 
comentaron que, si bien no hay diferencias entre niños y niñas en el logro en matemáticas, sí las hay en cómo lo alcanzan. Señalaron que mientras los niños emplean su talento "natural", las niñas tienen que desarrollar la habilidad para lograr los mismos resultados. Este tipo de afirmaciones pone en evidencia cómo las docentes proyectan su percepción de género en relación a las matemáticas (vistas como un dominio masculino) ya que, si bien no observan diferencias en el desempeño global, asumen que las niñas tienen que trabajar mucho más que los niños, quienes, según ellas, nacen con el talento para esta disciplina. Sin embargo, ante esta supuesta diferencia, que consideran de tipo genético, tratan de no ser indiferentes y desarrollan una estrategia de compensación para propiciar, según ellas, una mayor equidad de género, como se desprende del siguiente comentario de una docente:

"... Doy mayor preferencia a las niñas [en las interacciones que se dan en la clase de matemáticas] para compensar el talento de los niños en esta asignatura..."

“... para nivelar, cuando trabajamos en equipo siempre formamos equipos mixtos..."

La investigación también contempló observaciones de clase, en las cuales se detectaron interacciones de bajo y de alto nivel cognitivo (Wimer, Ridenour, Thomas y Place, 2001) dirigidas al grupo en general y a alumnos en particular. Las primeras, corresponden a interacciones que requieren que el estudiante evoque datos específicos, terminología, convenciones, clasificaciones, categorías, hechos y principios memorizados (por ejemplo: ¿Cuánto es $3 \times 7$ ?; ¿Qué es un triángulo?). Las interacciones de alto nivel cognitivo promueven el desarrollo de habilidades críticas del pensamiento (interpretación, extrapolación, aplicación, análisis, síntesis, evaluación), por ejemplo: ¿Cuántas veces saltó la pulga para igualar al conejo?; ¿Qué otro número sumado a este te puede dar 125? De acuerdo con su percepción de género y en aras de compensar las supuestas carencias "naturales" de las niñas y propiciar una mayor equidad, las maestras tendían a formular preguntas de alto nivel cognitivo sobre todo a los niños, mientras que las preguntas de bajo nivel cognitivo iban dirigidas preferentemente a las niñas.

El siguiente ejemplo ilustra esta tendencia:

Maestra: Juan, pasa por favor a explicar a tus compañeros cómo resolviste el problema [el problema era el siguiente: "En la aldea Panará, en Brasil, hay 16 chozas dispuestas en forma circular. En cada choza conviven dos familias de 5 miembros cada una. ¿Cuál es la población total de la aldea?"]

Maestra: Rosa ¿puedes pasar a resolver la operación? (en el pizarrón aparece la siguiente suma $326+507+135)$. 
De este modo, a través de un trato diferenciado entre niños y niñas, se observó que las docentes tenían expectativas de aprendizaje y académicas diferenciadas para cada uno de los sexos, colocándolos en esferas de capacidades diferentes, lo que, a la larga, puede tener consecuencias importantes en la autopercepción del alumnado con relación a las matemáticas. Como se puede observar, sin proponérselo ni tener conciencia de ello ("Mi organización y planeación de clases es asexuada") las maestras fomentaban y reforzaban las diferencias de género.

Ramírez (2006) concluye el estudio señalando que, si bien existe una creencia generalizada de que la escuela mixta proporciona a niños y niñas las mismas oportunidades de participar de las experiencias alfabetizadoras y numeradoras en el aula, esto no es así, ya que, como se pudo observar, se suele brindar mayores oportunidades a los niños, aunque de manera inconsciente y no reconocida por las docentes. Esto coloca a la escuela primaria, con la variable docente, como una generadora de las diferencias de género e inequidad en matemáticas que se van reforzando, más adelante, en la escuela secundaria, como podemos apreciar en el siguiente ejemplo.

\section{Un ejemplo en la escuela secundaria}

En una investigación realizada en 16 escuelas secundarias, técnicas y generales de Ciudad de México (Ursini, 2009) se entrevistó a 15 docentes de matemáticas responsables de los terceros años. Las entrevistas indagaron sobre su percepción de las diferencias de género en el logro matemático, en la participación en la clase de matemáticas y cuáles consideran que son las características de un estudiante que tiene éxito en matemáticas. Las respuestas pusieron en evidencia algunas de sus ideas acerca de la equidad de género en matemáticas (Ramírez y Chávez, 2009).

Si bien la mayoría del profesorado afirmó que no hay diferencia entre niñas y niños en la capacidad de aprender matemáticas, sus estereotipos de género se pusieron en evidencia cuando señalaron que las alumnas obtienen mejores calificaciones debido a ciertas características que consideraron propias del género femenino y no debido a sus capacidades intelectuales, como revelan los siguientes fragmentos:

“... las mujeres son 'macheteras', dedicadas, responsables, dinámicas, estudiosas, no faltan, son controlables, Ilegan temprano, sus trabajos son limpios, pulcros y sus participaciones y su comportamiento son buenos; que, si bien, las matemáticas les cuestan 'un poquito más de trabajo', logran llevar a cabo las tareas..."

"Los niños son más talentosos... las matemáticas son más de niños aunque las niñas también llegan a destacar en matemáticas con la práctica..." 
“...los niños son más talentosos en matemáticas, ya que son a quienes mandan a la tienda y a los mandados, además por su condición de hombre se les facilitan..."

“... bueno, les va mejor... a las mujeres les va mejor... porque se dedican más a estudiar o ponen más atención o tratan de ser más ordenadas en su cuaderno. Que en matemáticas tiene que ver demasiado el ser ordenado, el ser limpio, el poner atención y cuidado en un procedimiento. Las mujeres son más cuidadosas en eso..."

Según la mayoría de estos docentes a las chicas "... les cuesta más trabajo entender las matemáticas" y "tienen dificultades para aprobar los exámenes", por lo cual consideran necesario establecer algunas estrategias de compensación, como:

» Pedir más ejercicios y tareas y, al considerar que las mujeres son "... más ordenadas y responsables con las tareas que se solicitan en clase", esperan que las realicen y eso las ayuda a superar las dificultades que tienen "por naturaleza", esto es, debido a su sexo;

» evaluar no solo si contestaron correctamente las preguntas del examen, sino también otros aspectos, algunos de ellos no relacionados con contenidos matemáticos, como la asistencia, el comportamiento en clase, la presentación y entrega de tareas (estrategia legitimada por la errónea interpretación que hacen muchos profesores del Acuerdo Secretarial 592, que recomienda incorporar distintas técnicas e instrumentos en los procesos de evaluación, ello con dos intenciones: una, transformar las prácticas docentes; la otra, evitar la reprobación escolar).

Lo anterior se desprende del siguiente fragmento:

... la niña se dedica mucho a hacer los trabajitos, a hacerlos bien, a ponerles la florecita, a entregar, y los maestros evaluamos mucho eso, para secundaria, que cumplan. Si tú cumples con todo y sacas 3 en el examen, pasas. Cumples con tareas, con trabajos, no faltas, y no me das lata, aunque saques 2 o 3 en el examen a la hora del promedio vas a pasar, pero no ha llegado a los conocimientos, ¿sí? Los hombres son muy fodongones la mayoría, son muy fodongones: "Ahí le entrego en una hojita, le entrego acá...", pero parece ser que los conocimientos los tienen mejor establecidos y a la hora de ponerlos en práctica, y lo hacen...

Estas estrategias de compensación ponen de manifiesto la percepción diferenciada de género de la mayoría del profesorado. Sus comentarios muestran también que, a pesar de que admiten que los varones tienden a recibir peores calificaciones que las mujeres, están convencidos de que esto no se debe a la falta de conocimientos dado que, según ellos, los varones "los tienen mejor establecidos" que las mujeres, sino a que

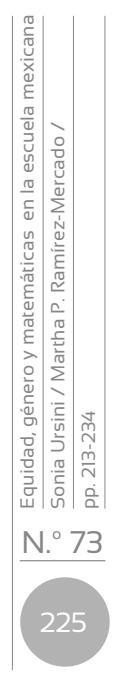


son más descuidados e incumplidos. Esta percepción de las capacidades de aprendizaje del alumnado, diferenciada por sexo, pone en evidencia una vez más los estereotipos de género presentes en muchos docentes, así como la visión de las matemáticas como un dominio masculino. En efecto, mientras perciben a las niñas como muy trabajadoras y estudiosas (las etiquetan de "macheteras"), atribuyen a los estudiantes varones mejores capacidades para captar los conceptos matemáticos, así como más confianza y seguridad en sus capacidades para llevar a cabo cualquier tarea de matemáticas, si bien reconocen que los varones son descuidados ("fodongos") en su elaboración y presentación. Al igual que las maestras de primaria, la mayoría del profesorado de secundaria entrevistado opinó que los alumnos varones tienen una capacidad "natural" para captar los conceptos matemáticos. Consideran que para ellos es suficiente poner atención a las explicaciones del profesor y no necesitan dedicar mucho tiempo al estudio, como es el caso de las niñas:

“Los niños captan, tienen habilidad para captar... pero se confían, ¿sí?

“... y sí, en cambio, la gran mayoría de los hombres tenemos esta característica auditiva, nada más con que le estemos poniendo atención, ya con eso... puesto que es lo que ha dado resultados, porque macheteros o tan concienzudos son pocos niños."

"Yo siento que es natural, es raro el hombre que es machetero, que se pasa las noches ahí, como la mujercita que está macheteando, macheteando, macheteando,..."

Esta concepción diferenciada de las capacidades de niños y niñas para trabajar en matemáticas junto con la intención del profesorado de propiciar una mayor equidad, se hace evidente también cuando afirman que, si bien "es de dominio público que los varones obtienen mejores calificaciones en los exámenes", ellos tratan de ayudar a las mujeres proporcionando guías de estudio "para todos, aunque sabemos que las únicas que las resuelven son las niñas... esto nos sirve para compensar". Consideran también necesario ponerles actividades más "a su alcance" ya que "las damitas tienen más dificultad, mientras a los varones se les facilita más, ya que son inteligentes". El profesorado no parece darse cuenta de que estas medidas compensatorias, lejos de propiciar más equidad fomentan la desigualdad.

La noción de equidad que se observa en el discurso del profesorado está cimentada en los estereotipos socioculturales de género, de acuerdo con los cuales se considera que los niños son prácticos, directos, capaces de poner atención y con una mayor capacidad de abstracción; en tanto que las niñas son ansiosas, nerviosas, intranquilas y faltas de carácter, si bien creativas. De ahí que consideren que para lograr una mayor equidad 
es necesario elaborar estrategias que permitan compensar las "debilidades femeninas naturales" para ayudarlas así a lograr, por lo menos aparentemente, un mejor desempeño matemático.

\section{La influencia de los estereotipos de género de los padres de familia}

En la investigación realizada por Ursini (2009) se aplicó un cuestionario dirigido a padres y madres de familia de estudiantes de escuelas secundarias públicas de Ciudad de México, con el propósito de conocer, entre otras cosas, a qué atribuían el logro o las dificultades de sus hijos en matemáticas.

Los resultados pusieron de manifiesto estereotipos de género similares a los ya señalados con relación al profesorado de matemáticas. Los padres de familia consideraron a las niñas cumplidas, trabajadoras, dedicadas, obedientes y disciplinadas, mientras calificaron a los varones como prácticos, inquietos, desobedientes y poco dedicados. Según la mayoría, las características atribuidas a las niñas, sobre todo el hecho de ser disciplinadas, son las que les permiten obtener buenas calificaciones "a pesar de ser niñas". Por el contrario, opinan que al no contar los varones con "buenos hábitos de estudio" ni con el apoyo suficiente por parte del "profesorado que apoya mucho más a las niñas", obtienen malas notas a pesar de tener una mayor capacidad "natural" para las matemáticas que, a su vez, asociaron con la inteligencia. Si bien los padres encuestados no asociaron explícitamente la inteligencia con un sexo en particular, en otro estudio Ursini y Sánchez (2008) encontraron que estudiantes de tercero de secundaria sí lo hacían. No se encontraron diferencias de género cuando señalaron que para aprender matemáticas es necesario prestar atención, tener interés, dedicación, disciplina, esforzarse y ser inteligente; sin embargo, estos factores se asociaron de manera diferenciada a hombres y a mujeres. Señalaron que para que los varones aprendan matemáticas es necesario que sean inteligentes, estén interesados en el tema y presten atención en clase, mientras que las mujeres necesitan dedicación, disciplina, esfuerzo y obedecer las consignas del profesor.

La influencia que ejerce sobre el alumnado el entorno familiar en la percepción de las matemáticas como un dominio masculino se hizo evidente también en los intercambios que se dieron en los grupos focales con alumnas y alumnos (Ursini, 2009). La gran mayoría manifestó, por ejemplo, que cuando necesitaban algún tipo de apoyo o explicación para comprender algún concepto matemático, resolver sus dudas o realizar sus tareas, acudían de preferencia a los hombres de la familia (preferentemente a sus padres, pero también a sus hermanos mayores, tíos, primos, cuñados)

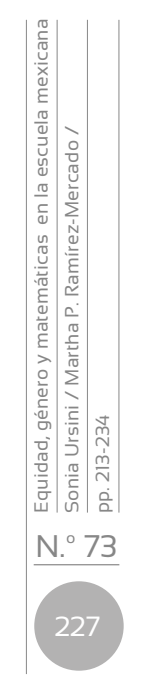


y no a sus madres u otras mujeres de la familia, dado que ellos son más comprensivos y dispuestos a apoyar, además suelen saber más matemáticas que ellas. Una niña, por ejemplo, comentó:

... mi mamá me regaña... ni entiendes, ni lo razonas bien... y como mi papá si sabe algo de matemáticas y todo eso, Ilego mejor con mi papá para que me explique y él me tiene más paciencia, me explica mejor...

De igual forma algunos estudiantes varones señalaron que en caso de necesidad suelen acudir a los hombres de la familia y no a las mujeres:

“... sí, mi hermano también me apoya..."

“... bueno mi papá es el que me puede ayudar..."

A través de los comentarios del estudiantado se pudo percibir también que las expectativas que tienen los padres de familia, en particular las madres, con respecto a los hijos varones y a las hijas mujeres son diferentes. Mientras que tratan de presionar a los varones para que aprendan matemáticas, con la idea de que eso puede mejorar sus oportunidades para realizar una carrera profesional ("... tienes que terminar una carrera y luego haces lo que tú quieras..."), en el caso de las hijas mujeres se limitan a pedirles que obtengan una calificación aprobatoria. Esta disparidad de expectativas de los padres de familia se manifiesta en los discursos de los y las estudiantes. Mientras los varones comentan que las matemáticas son una herramienta útil y probablemente necesaria para sus estudios futuros y otros aspectos de su vida, ellas aspiran a carreras que no impliquen muchas matemáticas o de plano las excluyan ("... me gustaría también ser educadora, sí, me encantan los niños chiquitos. Pero, todo, menos que sea algo de matemáticas"). En consonancia con los estereotipos de género dominantes, se inclinan por licenciaturas orientadas al cuidado o la atención de los otros (psicología, enfermería, magisterio), u oficios como estilista o azafata. Si bien consideran que pueden concluir una licenciatura, la asocian a tener una mejor calidad de vida en su rol de "madre-esposa-cuidadora":

“... Y pues, con mi propio dinero ayudar a mis papás, y pues estar en mi casa, y con hijos, tal vez, uno, o dos, o tres. Y obvio con esposo, si tengo hijos debo tener esposo ¿noooo?"

" ... Quiero tener hijos inteligentes y tener dinero, viviendo bien, con una carrera, familia, esposo, hijos, carrera." 


\section{Algunas reflexiones finales}

Analizar lo que sucede en la clase de matemáticas desde la perspectiva de género pone en evidencia varios de los factores que inciden en la creación y el reforzamiento de la inequidad de género en relación con la enseñanza y el aprendizaje de las matemáticas. Al analizar el ambiente que se crea en las clases de matemáticas de escuelas primarias y secundarias públicas en México, hemos podido constatar que siguen presentes algunos factores señalados desde los años ochenta y noventa en otros países. Se identificaron, además, otros factores, como las estrategias de compensación, a las que recurre el profesorado con el propósito de aminorar, desde su perspectiva, la falta de equidad de género. Estas estrategias responden al intento de cumplir con los requerimientos oficiales que solicitan al profesorado tomar medidas que propicien una mayor equidad de género. Al no existir indicaciones claras de qué implica este requerimiento, cada profesor, desde sus posibilidades y su idea de equidad, diseña estrategias que le permitan cumplir con las exigencias de las autoridades educativas. Sin embargo, no solo no logran su propósito, sino que, a la larga, refuerzan las desigualdades, además de poner en evidencia que las concepciones diferenciadas de género son muy profundas y arraigadas en nuestra sociedad. Sigue prevaleciendo, por ejemplo, la idea ya ampliamente desmentida por numerosas investigaciones y superada solo en pocos sectores de la sociedad, de que existen diferencias "naturales" debidas al sexo de la persona, con respecto a la capacidad de aprender matemáticas. A partir de este convencimiento, todavía muy arraigado, el profesorado establece interacciones diferenciadas entre los y las estudiantes, y planea y aplica estrategias de compensación diferenciadas. Se establecen así esferas de actuación para niños y niñas que requieren capacidades diferentes, sin tener conciencia plena de que con ello se fomentan y refuerzan las diferencias de género $y$, por tanto, la inequidad educativa. Esto afecta negativamente a las mujeres, dado que se les priva de la igualdad de oportunidades para acceder a la educación profesional y al campo laboral que requiere de conocimiento matemático.

Las ideas que dominan en nuestra sociedad acerca del rol fundamental de la mujer como reproductora de la especie y cuidadora de la familia llevan a los padres de familia a no generar las mismas expectativas en cuanto a la preparación matemática necesaria para sus hijos hombres y mujeres. Esto, junto con la baja expectativa que tiene el profesorado en la capacidad de las mujeres para aprender matemáticas, lleva a que se vaya propiciando y fortaleciendo en el estudiantado también la creencia de que los varones son "naturalmente" aptos para esta disciplina, mientras las mujeres no. Sigue predominando y se va perpetuando así la visión de las matemáticas como un dominio masculino.

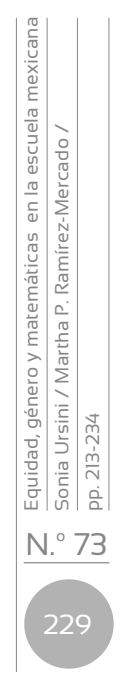


En la cotidianidad de la clase de matemáticas se dan tensiones complejas con respecto a la equidad de género, muchas de ellas imperceptibles para el profesorado y los padres de familia debido a la naturalidad con la que se conciben y justifican.

Las diferencias de género que se originan en la clase de matemáticas se deben a factores culturales y sociales, que apoyan la idea de un mundo organizado por características sexuales. Culturalmente, nuestra sociedad continúa promocionando estereotipos que no apoyan la construcción de la equidad de género en la clase de matemáticas. Es necesario, por lo tanto, transformar estos constructos culturales para poder generar nuevas formas de abordar la diversidad en la clase de matemáticas, que lleven a garantizar una mayor equidad de género.

El problema de la inequidad de género no es solo una cuestión de oportunidades de acceso de las mujeres a la educación. La equidad en la clase de matemáticas es mucho más que ofrecer las mismas oportunidades para acceder a este conocimiento. Si bien es necesario asegurar, en primera instancia, que tanto mujeres como hombres tengan igualdad de oportunidades formales y legales para acceder al conocimiento, esto no es suficiente. Es necesario que la sociedad en general y la comunidad escolar, en particular, tengan las mismas expectativas académicas, de aprendizaje y desarrollo profesional para ambos sexos.

Tanto la identidad diferenciada de género como la falta de equidad de género se han "naturalizado" e "invisibilizado" y permean profundamente nuestras sociedades, de ahí que abordar el problema de la equidad de género en matemáticas requiere de muchas y diversas aproximaciones, tanto teóricas como metodológicas, que permitan, en primera instancia, identificar y visibilizar la problemática, y ayuden a "afinar la mirada" para descubrir las sutiles formas a través de las cuales la inequidad en el género se va deslizando en nuestro sistema educativo.

\section{Referencias}

Barbieri, M. T. (1996). Certezas y malos entendidos sobre la categoría de género. Estudios básicos de Derechos Humanos IV, 47-84. Costa Rica: Instituto Interamericano de Derechos Humanos,

Bolívar, A. (2005). Equidad educativa y teorías de la justicia. Reice-Revista Electrónica Iberoamericana sobre Calidad, Eficacia y Cambio en Educación, 3(2), 42-69.

Burton, L. (1986). Girls into mathematics can go. Londres: Holt, Rinehart and Winston.

Burton, L. (1990). (Ed.). Gender and mathematics: An international perspective. Londres: Cassell. 
Bustos, O. (2001). Género y socialización: familia, escuela y medio de comunicación. En M. A. González Pérez y J. Mendoza-García (comps.). Significados colectivos: procesos y reflexiones teóricas (pp. 289-358). México: Tecnológico de Monterrey/Ciiacso,

Bustos, O. (1994). La formación del género: el impacto de la socialización a través de la educación. En Conapo, Antología de la sexualidad humana (pp. 389-425). México: Conapo-Porrúa.

Campos, C. (2006). Actitud hacia las matemáticas: diferencias de género entre estudiantes de sexto de primaria y tercer grado de secundaria (tesis inédita de maestría). Cinvestav-IPN, México.

Chávez, S. C. (2013). Cinvestav. Escasa participación femenil mexicana en ciencia. Alianzatex. marzo 07, 2013. Recuperado el 11 de octubre de 2016, de http://www.alianzatex.com/nota.php?nota=N0022948

Connell, R.W. (1997). Escuelas y justicia social. España: Morata.

Emmelhainz, I. (2016). La tiranía del sentido común. La reconversión neoliberal de México. México: Paradiso.

Ernest, J. (1980). Is mathematics a sexist discipline? En L. Fox, L. Brody, D. Tobin (eds.). Women and mathematical mystique. Proceeding of the Eighth Annual Hymen Blumberg Symposium on Research in Early Childhood Education (pp. 7-22). Estados Unidos: Johns Hopkins University.

Eudave, M. (1994). Las actitudes hacia las matemáticas de los maestros y alumnos de bachillerato. Educación Matemática, 6(1), 46-58.

Fennema, E. (1974). Mathematics learning and the sexes: A review. Journal for Research in Mathematics Education, 5(3), 126-139. Doi: $10.2307 / 748949$

Fennema, E. y Sherman, J. (1976). Fennema-Sherman Mathematics Attitudes Scale: Instruments designed to measure attitudes toward the learning of mathematics by males and females. JSAS Catalog of Selected Documents in Psychology, 6, 31 (Ms. 1225). Journal for Research in Mathematics Education, 7, 324-326.

Fennema, E. y Sherman, J. (1978). Sex-related differences in mathematics achievement and related factors: A further study. Journal for Research in Mathematics Education, 9, 189-203.

Flores-Palacios, F. (2001). Psicología social y género. El sexo como objeto de representación social. México: McGraw-Hill.

Flores, R. (2007). Representaciones de género de profesores y profesoras de matemáticas, y su incidencia en los resultados académicos de alumnos y alumnas. Revista Iberoamericana de Educación, 43(1), 103-118.

González, J. R. (2003). Diferencias de género en el desempeño matemático de estudiantes de secundaria. Educación Matemática, 15(2), 129-161.

Guarro, A. (2002). Currículum y democracia. España: Octaedro.

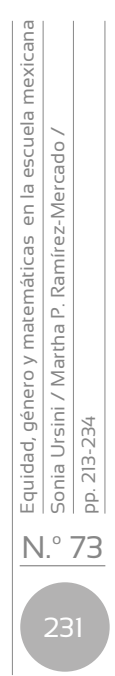


Hanna, G. (2003). Reaching gender equity in mathematics education. The Educational Forum. Doi: 10.1080/00131720309335034

Hyde, J. S.; Fennema, E. y Lamon, S. J. (1990). Gender differences in mathematics performance. Psychological Bulletin, 107, 139-155.

Lamas, M. (1986). La antropología feminista y la categoría de género. Nueva Antropología. Estudios sobre la mujer, problemas teóricos. NAH. Revista de Ciencias Sociales, 7(30), 173-198.

Morales, C. (1998). Actitudes de los escolares hacia la computadora y los medios para el aprendizaje. Tecnología y Comunicación Educativa, 28, 51-65.

Ramírez, M. (2006). Influencia de la visión de género de las docentes en las interacciones que establecen con el alumnado en las clases de matemáticas (tesis inédita de Maestría). Cinvestav-IPN, México.

Ramírez, M. P. y Chávez, Y. (2011). Conceptualizaciones de docentes y directivos de las matemáticas en educación secundaria. Un estudio de género en el D. F. Acta Latinoamericana de Matemática Educativa, 24, 229-240.

Rascón, M. (2000). El proceso de identificación femenina y su relación con el imaginario social (Tesis de Maestría en Psicología Clínica). unam Conacit/uam, Iztapalapa.

Rawls, J. (1979). Teoría de la justicia. Madrid: Fondo de Cultura Económica.

Real, C. y Ursini, S. (2010). Gender differences when working with algebraic variables: A study with Mexican secondary school students. En Forgasz, Becker, Lee y Steinthorsdottir (ed.). International perspectives on gender and mathematics education. (173-202).

Rivas, A. (2004). Gobernar la educación: estudio comparado sobre el poder y la educación en las provincias argentinas. Argentina: Granica.

Rivera, S. (2003). Diferencia de género en la visualización espacial; un estudio exploratorio con estudiantes de $2^{\circ}$ de secundaria (tesis inédita de aestría). Cinvestav-IPN:México.

Rodríguez, C. y Ursini, S. (2008). Social representation and gender in the teaching of mathematics with multimedia devices. 11th International Congress of Mathematics Education (ICME, 11), Topic Study Group 32: Gender and mathematics education, Monterrey, México. Recuperado el 6 de junio de 2009, de http//tsg.icme11.org/document/get/166.

Rossi Baker, J. (1981). Differential treatment of female and males in mathematics classes. Journal for Research in Mathematics Education, 12(1), 40-53.

Scott, J. (1986). Gender: A useful category of historical analysis. American Historical Review, 91, 1053-1075. 
Secretaría de Educación Pública (2011). Acuerdo Secretarial 592 por el que se establece la articulación de la Educación Básica en México. México.

Sherman, J. y Fennema, E. (1978). Sex-related differences in mathematics achievement and related factors: A further study factors. Journal for Research in Mathematics Education, 9(3), 189-203.

Spencer, D. y Sarah, E. (1993). Aprender a perder. El sexismo en la escuela. España: Paidós.

Subirat, M. y Brullet, C. (1997). Rosa y azul. La transmisión de los géneros en la escuela mixta. En ... Otra forma de ser maestra, madres y padres... (pp. 101-114). Grupo de Educación Popular con Mujeres. México.

Tiedemann, J. (2002). Teachers' gender stereotypes as determinants of teacher perceptions in elementary school mathematics. Educational Studies in Mathematics, 50(1), 49-62.

Ursini, S. (2009). Aspectos educativos y género en el aprendizaje de las matemáticas en escuelas secundarias del Distrito Federal. Cuaderno de Trabajo 15. México: Instituto Nacional de las Mujeres.

Ursini, S. (2010). Diferencias de género en la representación social de las matemáticas: un estudio con alumnos de secundaria. En Blázquez, Flores-Palacios y Ríos (eds.). Investigación feminista, epistemología, metodología y representaciones sociales (379-398). México. Coedición UNAM, CEIICH, CRIM y Facultad de Psicología unam. Colección Debate y Reflexión.

Ursini, S. (2013). Las diferencias de género en matemáticas: una realidad poco atendida desde las representaciones sociales. En Flores-Palacios (ed.). Representaciones sociales y contextos de investigación con perspectiva de género (pp. 123-142). México: CRIM-UNAM-Conacyt.

Ursini, S.; Ramírez, M. P. y Sánchez, G. (2007). Using technology in the mathematics class: How this affects students' achievement and attitudes. Proceedings of the 8th Iстмт (Integration of ICT into Learning Processes) (p. 29) [CD-ROM]. University of Hradec Králové, Czech Republic.

Ursini, S.; Sánchez, G.; Orendain, M. y Butto, C. (2004). El uso de la tecnología en el aula de matemáticas: diferencias de género desde la perspectiva de los docentes. Enseñanza de las Ciencias, 22(3), 409-424.

Ursini, S. y Sánchez, G. (2008). Gender, technology and attitude towards mathematics: a comparative longitudinal study with Mexican students. ZDM, Mathematics Education 40(4), 559-577. DOI: 10.1007s11858-008-01-20-1. 
Wimer, J.; Ridenour, C.; Thomas, K. y Place, W. (2001). Higher order teacher questioning of boys and girls in elementary mathematics classrooms. The Journal of Educational Researches, 95(2), 84-91.

Wrigley, J. (1992). Gender and education in the Welfare State. En J. Wrigley (ed.). Education and gender equality (pp. 1-249). Gran Bretaña: Burgess Science Press. 